\title{
Protective effects of calorie restriction on insulin resistance and islet function in STZ-induced type 2 diabetes rats
}

Li Zhang ${ }^{1,2+}$, Ying-juan Huang ${ }^{2+}$, Jia-pan Sun ${ }^{2}$, Ting-ying Zhang ${ }^{1}$, Tao-li Liu' ${ }^{1}$, Bin Ke ${ }^{3}$, Xian-fang Shi ${ }^{1}$, Hui Li Geng-peng Zhang ${ }^{1}$, Zhi-yu Ye', Jianguo Hu' and Jian Qin ${ }^{1 *}$ (D)

\begin{abstract}
Background: Caloric restriction (CR) has become increasingly attractive in the treatment of type 2 diabetes mellitus (T2DM) because of the increasingly common high-calorie diet and sedentary lifestyle. This study aimed to evaluate the role of CR in T2DM treatment and further explore its potential molecular mechanisms.

Methods: Sixty male Sprague-Dawley rats were used in this study. The diabetes model was induced by 8 weeks of high-fat diet (HFD) followed by a single dose of streptozotocin injection $(30 \mathrm{mg} / \mathrm{kg})$. Subsequently, the diabetic rats were fed HFD at $28 \mathrm{~g} /$ day (diabetic control) or $20 \mathrm{~g} /$ day (30\% CR regimen) for 20 weeks. Meanwhile, normal rats fed a free standard chow diet served as the vehicle control. Body mass, plasma glucose levels, and lipid profiles were monitored. After diabetes-related functional tests were performed, the rats were sacrificed at 10 and 20 weeks, and glucose uptake in fresh muscle was determined. In addition, western blotting and immunofluorescence were used to detect alterations in AKT/AS160/GLUT4 signaling.

Results: We found that 30\% CR significantly attenuated hyperglycemia and dyslipidemia, leading to alleviation of glucolipotoxicity and thus protection of islet function. Insulin resistance was also markedly ameliorated, as indicated by notably improved insulin tolerance and homeostatic model assessment for insulin resistance (HOMA-IR). However, the improvement in glucose uptake in skeletal muscle was not significant. The upregulation of AKT/AS160/GLUT4 signaling in muscle induced by 30\% CR also attenuated gradually over time. Interestingly, the consecutive decrease in AKT/AS160/GLUT4 signaling in white adipose tissue was significantly reversed by $30 \%$ CR.
\end{abstract}

Conclusion: CR (30\%) could protect islet function from hyperglycemia and dyslipidemia, and improve insulin resistance. The mechanism by which these effects occurred is likely related to the upregulation of AKT/AS160/GLUT4 signaling.

Keywords: Calorie restriction, Diabetes, Insulin resistance, Streptozotocin, AKT /AS160/GLUT4

\section{Introduction}

Type 2 diabetes mellitus (T2DM) is a metabolic disorder, the occurrence and progression of which are closely

\footnotetext{
*Correspondence: himybox@yeah.net

${ }^{\dagger}$ Li Zhang and Ying-juan Huang contributed equally to this work.

1 Department of Traditional Chinese Medicine, The Seventh Affiliated Hospital, Sun Yat-Sen University, Shenzhen 518107, China

Full list of author information is available at the end of the article
}

related to individual lifestyles and other risks, such as genetic factors [1]. Caloric restriction (CR), the only approved scientific method that can slow aging, has been proven to improve insulin resistance (IR) and islet dysfunction. Moreover, a recent clinical study [2] suggested the potential of a very low-calorie diet for the remission of T2DM. However, the molecular mechanisms underlying $\mathrm{CR}$ in T2DM treatment remain unclear.

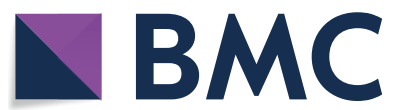

(c) The Author(s) 2021. Open Access This article is licensed under a Creative Commons Attribution 4.0 International License, which permits use, sharing, adaptation, distribution and reproduction in any medium or format, as long as you give appropriate credit to the original author(s) and the source, provide a link to the Creative Commons licence, and indicate if changes were made. The images or other third party material in this article are included in the article's Creative Commons licence, unless indicated otherwise in a credit line to the material. If material is not included in the article's Creative Commons licence and your intended use is not permitted by statutory regulation or exceeds the permitted use, you will need to obtain permission directly from the copyright holder. To view a copy of this licence, visit http://creativecommons.org/licenses/by/4.0/. The Creative Commons Public Domain Dedication waiver (http://creativeco mmons.org/publicdomain/zero/1.0/) applies to the data made available in this article, unless otherwise stated in a credit line to the data. 
The insulin signaling pathway is crucial for normal insulin action. In skeletal muscle or white adipose tissue, activated AKT phosphorylates the $160-\mathrm{kDa}$ substrate of AKT (AS160) to block the inactivation of small Rab GTPase protein switches that control vesicle trafficking. This process facilitates the translocation of glucose transporter 4 (GLUT4), further mediating glucose uptake in these tissues to maintain glucose homeostasis [3]. Notably, apart from insulin-induced glucose uptake, most of the other effects of insulin are retained or only slightly affected in individuals with IR. IR can be defined as a decrease in insulin-induced glucose uptake [3]. Therefore, normal transduction of AKT/AS160/GLUT4 signaling in skeletal muscle or white adipose tissue is critical to guarantee well-balanced glucose uptake to maintain normal insulin sensitivity. It has been reported that the phosphorylation level of AKT in patients with IR decreased by up to $50 \%$ when compared with that in healthy controls [4-6]. Muscle and adipose tissue-specific GLUT4 knockout can induce global IR and hyperinsulinemia [7, 8]. Mutations in AS160 also lead to IR and an increased risk of progression to T2DM in humans [9].

Based on the aforementioned information, AKT/ AS160/GLUT4 signaling may play an important role in the onset of IR and T2DM. Fortunately, the positive effects of CR on AKT and its phosphorylation have been observed in many studies [10-12], and several preliminary studies have reported the potential of CR in regulating GLUT4 $[13,14]$. Thus, we speculated that the mechanism underlying the metabolic effects of $\mathrm{CR}$ in T2DM treatment may be relate to its regulation of AKT/AS160/GLUT4 signaling. T2DM model rats were induced by feeding high-fat diet (HFD) combined with a single low dose of streptozotocin (STZ) injection, and then maintained on a $30 \%$ CR regimen to test our hypotheses.

\section{Methods and materials Materials}

Gansulin-R was purchased from Tonghua Dongbao Pharmaceutical Co. (Beijing, China). STZ and all other chemicals were purchased from Sigma-Aldrich (St. Louis, MO, USA) unless stated otherwise. Cell Counting Kit-8 (CCK-8) was purchased from Dojindo Laboratories (Kumamoto, Japan). The rat insulin ELISA assay was purchased from Mercodia (Mercodia AB, Uppsala, Sweden). The primary and secondary antibodies used in the experiments are summarized in table (Additional file 1: Table 1).

\section{Experimental procedures Animals}

A total of 60 weaned and specific-pathogen-free SD rats (male, 6-8 weeks, 180-190 g) were obtained from the Experimental Animal Center of Sun Yat-sen University (Guangzhou, China). The animal experiments were performed according to internationally followed ethical standards and approved by the research ethics committee of the School of Public Health, Sun Yat-sen University (no. 2019-001). Rats were kept on a $12 \mathrm{~h} \mathrm{light/dark}$ cycle in a temperature-controlled room maintained at $(24 \pm 1){ }^{\circ} \mathrm{C}$. with a relative humidity of $(50 \pm 5) \%$ and were maintained on a standard chow diet. Rats were habituated to the conditions for 1 week before modeling.

\section{Induction of diabetes}

With reference to the method widely used in the existing literature [15-17], rats were fed HFD for 8 weeks and then given a single low-dose of injection of STZ (30 mg/ $\mathrm{kg}$ ) to induce the T2DM model. Specifically, rats were randomly allocated to the blank control group $(n=20)$ and the model group $(n=40)$ according to their body mass. Rats in the blank control group had a free normal diet (ND, Guangdong Medical Laboratory Animal Center, Foshan, China; containing 58\% carbohydrates, $18 \%$ protein, $4.5 \%$ fat, and $4 \%$ essential vitamins and trace elements, with a total of $3.45 \mathrm{kcal} / \mathrm{g}$ ). Rats in the model group were fed HFD (Guangdong Medical Laboratory Animal Center, Foshan, China; containing 53.6\% normal chow, $15 \%$ sucrose, $10 \%$ lard, $20 \%$ protein, $1.2 \%$ cholesterol, and $0.2 \%$ sodium cholate, with a total of $4 \mathrm{kcal} / \mathrm{g}$ ). After 8 weeks of feeding, rats in the model group were intraperitoneally injected with $1 \% \mathrm{STZ}$ dissolved in $0.1 \mathrm{M}$ citric acid buffer $(\mathrm{pH}=4.2-4.5)$ at a single dose of $30 \mathrm{mg} /$ $\mathrm{kg}$, while the blank control rats received the same amount of citric acid buffer $(0.1 \mathrm{M})$. It is important to note that light had to be avoided throughout the entire procedure of preparation and injection of STZ solution, and the dissolved STZ solution was used as quickly as possible (within $15 \mathrm{~min}$ ). After STZ administration, model rats were provided with HFD ad libitum in order to attain a relatively stable blood glucose level. Fourteen days after STZ injection, random blood glucose (RBG) was measured with a portable glucometer (Beijing Yicheng biotechnology co. LTD, China) through the tail vein. Rats with RBG levels higher than or equal to $16.7 \mathrm{mmol} / \mathrm{L}$ were considered as diabetic [18] and were further used in the following experiment. 


\section{CR procedure}

During HFD feeding, the total food intake of rats in each cage was recorded to calculate the average daily food intake per rat, which was $28.54 \pm 4.78$ g/day. Thus, $30 \%$ CR (20 g/day) was used in the following experiments. The diabetes model was successfully induced in a total of 29 rats after STZ administration. Thereafter, according to blood glucose level, diabetic rats were randomly assigned to the model control group provided HFD ad libitum with high-fat diet (HFD + AL + STZ, MCT group, $\mathrm{n}=15$ ) or the CR intervention group fed a HFD with a $30 \%$ CR regimen, as stated above (HFD $+\mathrm{CR}+\mathrm{STZ}, \mathrm{CR}$ group, $\mathrm{n}=14$ ). Meanwhile, rats in the blank control group continued to be provided free access to normal diet $(\mathrm{ND}+\mathrm{AL}, \mathrm{BCT}$ group, $\mathrm{n}=20)$. The intervention period lasted for 20 weeks. Water was provided to all rats ad libitum throughout the experiment. The entire process of this experiment is detailed in Fig. 1.

Body mass and water intake were recorded every week, while RBG and fasting blood glucose (FBG) levels were measured every 2 or 4 weeks respectively. To assess insulin resistance, and insulin tolerance test (ITT) was conducted at baseline before modeling, after modeling, and at 10 and 20 weeks post-treatment. In addition, FBG and fasting insulin (FINS) were also detected at the indicated time points through the tail vein after a 12-h overnight fasting period, and insulin levels were measured by ELISA according to the manufacturer's instructions. An intraperitoneal glucose tolerance test (IPGTT) and glucose-stimulated insulin secretion (GSIS) were performed after modeling (before intervention), and at 10 and 20 weeks after intervention to evaluate glucose tolerance and islet secretion.

At 10 weeks and 20 weeks after intervention, 6-10 rats in each group were anesthetized with $2 \%$ pentobarbital sodium $(60 \mathrm{mg} / \mathrm{kg})$ after overnight fasting $(12 \mathrm{~h})$, and then blood, liver, pancreas, skeletal muscle, and white adipose tissue (WAT, specifically epididymal fat pad) were rapidly collected. Blood samples were centrifuged at $4{ }^{\circ} \mathrm{C}$ and $3500 \mathrm{rpm}$ for 15 min to collect the serum and stored at $-80{ }^{\circ} \mathrm{C}$ until use. The lipid profile was measured in the central laboratory of the First Affiliated Hospital of Sun Yat-sen University.

\section{IPGTT}

Rats were intraperitoneally injected with $50 \%$ glucose solution $(2 \mathrm{~g} / \mathrm{kg})$ after overnight fasting for $12 \mathrm{~h}$. Blood glucose was measured before injection $(0 \mathrm{~min})$ and 15 , 30, 60, 90, and 120 min after injection through the tail vein with a portable glucometer. Six rats from each group were randomly selected for the study.
ITT

Rats were intraperitoneally injected with Gansulin$\mathrm{R}(0.5 \mathrm{IU} / \mathrm{kg})$ after fasting for $6 \mathrm{~h}$. Blood glucose was measured before injection ( $0 \mathrm{~min}$ ) and 15, 30, 45, 60 and 90 min after injection through the tail vein with a portable glucometer. Six rats from each group were randomly selected for the study.

\section{GSIS}

Rats were intraperitoneally injected with $50 \%$ glucose solution $(2 \mathrm{~g} / \mathrm{kg})$ after $12 \mathrm{~h}$ of fasting at night. Blood samples were collected from the tail vein before injection (0 min) and 15, 30 and $60 \mathrm{~min}$ after injection. The blood samples were centrifuged at $3500 \mathrm{rpm}, 4{ }^{\circ} \mathrm{C}$ for $15 \mathrm{~min}$. The separated serum was collected and the insulin level was detected by ELISA. Six rats from each group were randomly selected for the study.

\section{Glucose uptake in isolated soleus}

At the end of the experiment, glucose uptake in the isolated soleus was measured in four rats from each group. The effect of CR on glucose uptake was detected according to the previous studies, with slight modifications [1921]. Generally, the isolated fresh soleus was immediately rinsed with Krebs-Ringer buffer, and the muscle fascia, tendons and attached connective tissue were removed as much as possible. Subsequently, the muscle tissue was placed into the brain slice mold (Reward, Shenzhen, China) to cut it into thin slices of similar size with a blade, then divided into three parts (roughly the same weight) and placed into separate $2 \mathrm{~mL}$ Eppendorf tubes. The muscle tissue was then incubated with $1.5 \mathrm{~mL}$ oxygenated glucose-free Krebs-Henseleit buffer (KRH) containing $0.1 \%$ bovine serum albumin (BSA) and $10 \mathrm{mU} /$ $\mathrm{mL}$ insulin (Gansulin-R) at $37^{\circ} \mathrm{C}$ and $300 \mathrm{rpm}$ (shaking) for $60 \mathrm{~min}$ to deplete intercellular glucose. After that, the liquid was removed, oxygenated with KRH $(1.5 \mathrm{~mL}$, containing $11.1 \mathrm{mM}$ glucose, $0.1 \% \mathrm{BSA}$, and $40 \mathrm{mM}$ mannitol) with 0,10 , or $20 \mathrm{mU} / \mathrm{mL}$ insulin. Another tube contained an equal volume of oxygenated $\mathrm{KRH}$, but no muscle tissue served as a blank control. All the four tubes were incubated at $37^{\circ} \mathrm{C}$ and $300 \mathrm{rpm}$ (shaking) for $30 \mathrm{~min}$. A $1 \mathrm{~mL}$ aliquot was collected from each incubation tube before and after incubation, and the glucose concentration was determined. Glucose uptake of muscle was calculated as the amount of glucose $(\mathrm{mg})$ taken up per gram of muscle tissue using the following formula: muscle glucose uptake $=\left(G_{b}-G C_{\mathbf{a}}\right) /$ muscle tissue weight, where $G C_{b}$ and $G C_{a}$ are the glucose concentrations before and after incubation, respectively. 


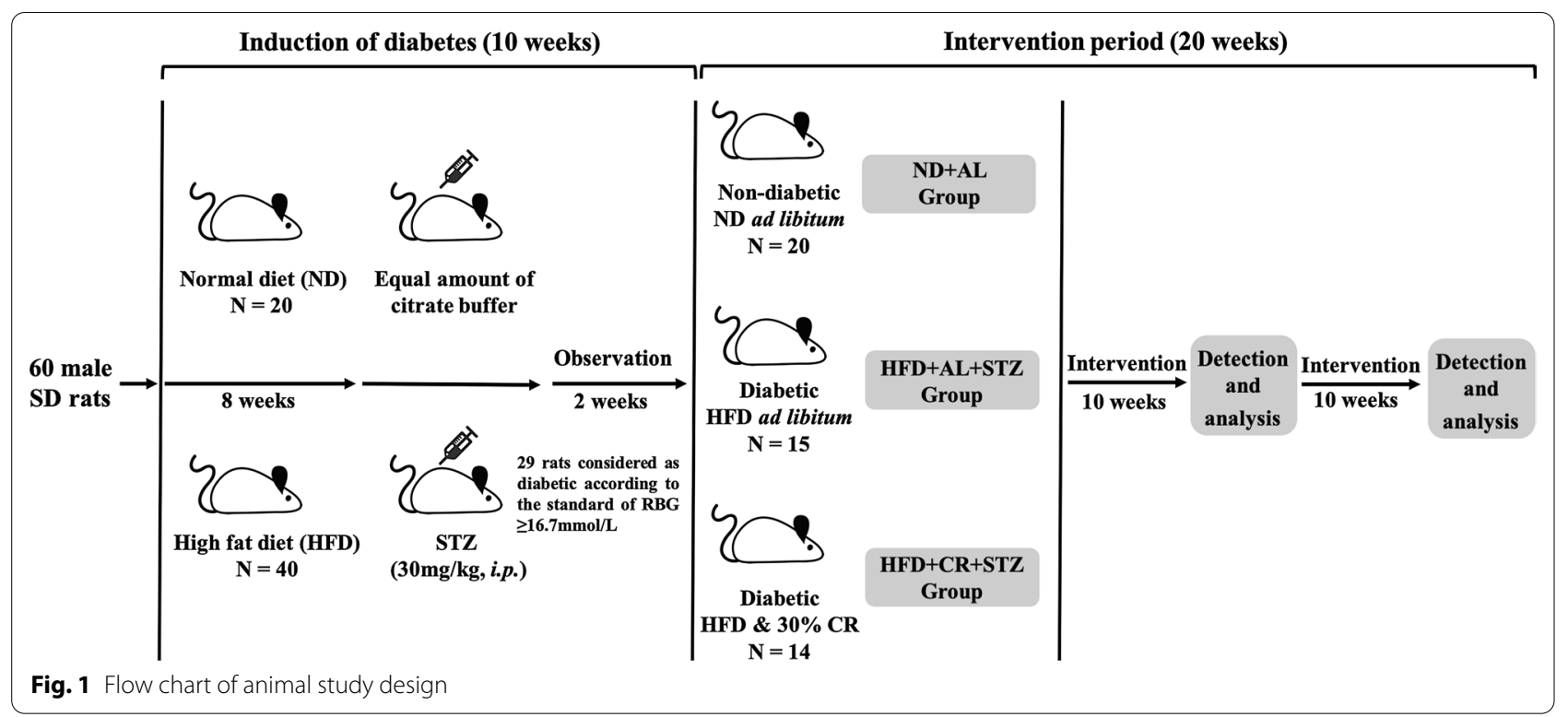

\section{Immunofluorescence}

Skeletal muscle from three rats in each group was randomly selected for GLUT4 immunofluorescence experiments. Briefly, after fixation in $4 \%$ paraformaldehyde overnight at $4{ }^{\circ} \mathrm{C}$, the muscle tissues were equilibrated in $20 \%$ sucrose overnight at $4{ }^{\circ} \mathrm{C}$ until the tissues settled to the bottom. The tissues were then transferred into 30\% sucrose overnight at $4{ }^{\circ} \mathrm{C}$ until they settled to the bottom, after which they were embedded in OCT for frozen sectioning. Sections were sliced at a thickness of $10 \mu \mathrm{m}$. Subsequently, frozen sections were rinsed gently with $4{ }^{\circ} \mathrm{C}$ phosphate-buffered saline (PBS) three times, fixed with $4{ }^{\circ} \mathrm{C} 4 \%$ PFA for $30 \mathrm{~min}$ at room temperature, and then washed three times with PBS again. After incubation with $0.2 \%$ Trition-X100 in PBS for $20 \mathrm{~min}$, sections were blocked with $3 \%$ BSA for $30 \mathrm{~min}$ at room temperature and then incubated overnight with rabbit anti-GLUT4 antibody (1:250, Abcam, ab654, Cambridge, MA, USA) at $4{ }^{\circ} \mathrm{C}$ in a wet box. After washing with Tris-buffered saline containing $0.1 \%$ Tween 20 , the sections were incubated with donkey secondary antibody conjugated with Alexa Fluor $^{\circledR} 568$ (1:400, Invitrogen, A-11011, Carlsbad, CA, USA) for $3 \mathrm{~h}$ at room temperature. Nuclei were stained with 4',6-diamidino-2-phenylindole (DAPI, $1 \mu \mathrm{g} / \mathrm{mL}$, Sigma-Aldrich, St. Louis, MO, USA). All photomicrographs were taken using an inverted fluorescence microscope (Nikon Eclipse Ti-E, Nikon, Japan).

\section{Western blotting}

Total protein extracts of skeletal muscle and epididymal fat pad tissues were obtained by homogenizing and lysing with RIPA buffer (Beyotime, Shanghai, China), followed by centrifugation at $14,000 \times g$ for 5 min at $4{ }^{\circ} \mathrm{C}$. Protein concentration was measured using a BCA assay kit (Beyotime, Shanghai, China). Equal amount of protein $(20 \mu \mathrm{g})$ were loaded into each lane. Proteins were separated by sodium dodecyl sulfate-polyacrylamide gel electrophoresis and electrically transferred to a polyvinylidene difluoride membrane (Millipore, Boston, MA, USA). After blocking the membrane with $5 \%$ skim milk, the target proteins were immunodetected using specific antibodies. After incubation with the secondary antibody, bands were visualized using an ECL plus kit (ThermoFisher, Waltham, MA, USA) and exposed to autoradiographic films according to the manufacturer's instructions. The intensities of bands were determined using ImageJ 1.52q software.

\section{Statistical analysis}

Statistical analysis was performed using SPSS 23.0 software. Data are presented as the mean \pm standard deviation (SD), and statistical significance was determined using one-way analysis of variance (ANOVA) followed by multiple comparisons with Tukey's test. Statistical significance was set at $p<0.05$.

\section{Results}

A total of 40 rats received STZ injection after 8 weeks of HFD feeding, among which 29 attained targeted RBG levels $(\geq 16.7 \mathrm{mmol} / \mathrm{L})$ and were used in the following experiment. Five rats died within 3 days of STZ administration because of intolerance to STZ toxicity. Furthermore, RBG concentrations of another 6 rats 
fluctuated in the range of $5.5-9.8 \mathrm{mmol} / \mathrm{L}$, and were therefore excluded from the following experiment. During the entire intervention period, there were no death in the blank control group (ND+AL). However, two rats in the MCT group (HFD+AL +STZ) died within 6 weeks, probably due to higher blood glucose levels (fluctuated in 22-27 mmol/L) and lower body weight, which influenced their acquisition of food and water. One rat in the CR group (HFD + CR + STZ) died accidentally from excessive anesthesia with isopentane inhalation when drawing blood from the tail vein in the GSIS experiment.

\section{Effects of CR on body mass and glucolipotoxicity}

Body weight at each time point in the $\mathrm{CR}$ group was lower than that in the MCT group, but the difference was not statistically significant (Fig. 2a, b, $p>0.05$ ). However, when compared with the baseline, a significant decrease was observed in the CR group (Fig. 2a, b, $p<0.05$ ) but not in the MCT group (Fig. 2a, b, $p>0.05$ ). Meanwhile, water intake in the MCT group increased gradually, while it declined to the blank control level after 20 weeks of CR intervention (Fig. 2c, d, $p>0.05$ ).

During the intervention period, RBG and FBG levels increased significantly in the MCT group and decreased gradually in the CR group. Ultimately, hyperglycemia was ameliorated by 20 weeks of CR intervention. (Fig. 2e, f, $p<0.05)$. Meanwhile, improvement in lipid profile including free fatty acids (FFAs, Fig. $2 \mathrm{k}, p<0.05$ ) was also detected after CR intervention (Additional file 1: Fig. 1). These results demonstrated that $\mathrm{CR}$ intervention provide relief from glucolipotoxicity. Accordingly, we found that glucose tolerance in the CR group at 20 weeks was significantly improved when compared to that at baseline or in the MCT group. (Fig. 2i, j, $p<0.05$ ). The area under the curve (AUC) of the IPGTT also showed a similar variation tendency (Fig. 2 j, $p<0.05$ ).

\section{Effects of $\mathrm{CR}$ on insulin resistance and islets function}

After 20 weeks of intervention, insulin sensitivity was notably improved in the CR group compared with that in the MCT group, as indicated by the ITT results. (Fig. 3a, $p<0.05$ ). Interestingly, homeostatic model assessment for insulin resistance (HOMA-IR) values in the $\mathrm{CR}$ and MCT groups both exhibited a significant decreasing tendency, and were significantly lower than their baseline values (Fig. $3 \mathrm{~d}, p<0.05$ ). Glucosestimulated insulin secretion levels in the CR and MCT groups were both much lower than those in the BCT group (Fig. 3b, c, $p<0.05$ ). Nevertheless, GSIS gradually improved in the $\mathrm{CR}$ group and progressively deteriorated in the MCT group over time (Fig. 3b, c). Insulin levels and AUC of GSIS in the CR group at 20 weeks were significantly higher than those in the baseline and the MCT group, although they were still markedly lower than those in the BCT group (Fig. 3b, c, $p<0.05$ ).

\section{Effects of $\mathrm{CR}$ on glucose uptake in skeletal muscle}

The glucose uptake rate of muscle in the $\mathrm{BCT}$ group increased proportionally with the elevation of insulin concentration applied for stimulation, while this effect was distinctly impaired in both the CR and MCT groups (Fig. 3e). Although the glucose uptake rate in the CR group at each insulin concentration was slightly higher than that in the MCT group, the difference was not statistically significant (Fig. 3e, $p>0.05$ ).

\section{Effects of CR on AKT/AS160/GLUT4 signaling}

In skeletal muscle, $C R$ intervention could exert protective effects on the greatly reduced expression of AKT, p-AKT, AS160, and GLUT4 proteins induced by T2DM pathology and HFD feeding. However, the protective effects of CR wore off over time. The expression of the aforementioned proteins and the average fluorescence intensity of GLUT4 decreased to the same levels as the MCT group at 20 weeks (Fig. 4a-c, Fig. 5, $p>0.05$ ). In contrast, in white adipose tissue, the protein expression of AKT, p-AKT, AS160, and GLUT4 in the MCT group decreased sharply over time, and were markedly lower than those in the CR groups at 20 weeks (Fig. $4 \mathrm{~d}-\mathrm{f}, p<0.05$ ).

\section{Discussion}

In a diabetic rat model induced by HFD feeding combined with a single low-dose injection of STZ, we demonstrated that $30 \%$ CR could protect islet function against glucolipotoxicity during the progression of T2DM by markedly relieving hyperglycemia and dyslipidemia. CR also improved insulin resistance significantly, with probable underlying molecular mechanisms related to the upregulation of AKT/AS160/GLUT4 signaling.

$\mathrm{CR}$ has attracted increasing attention in the treatment of metabolic diseases, as overeating and obesity are increasingly common in modern society. Therefore, weight loss has always been considered as the main effect of $C R$ and the source of its other accompanying metabolic benefits. However, some studies [22, 23] have indicated that weight loss may not be the only key function of CR in improving glucose metabolism. In our study, hyperglycemia in MCT rats deteriorated over time, resulting in aggravated osmotic diuresis, massive loss of glucose, and subsequent continuous weight loss. In contrast, diabetic rats receiving $\mathrm{CR}$ intervention experienced great relief from hyperglycemia, protecting them from continuous weight loss. However, insufficient calorie intake might offset the effect of weight gain resulting from hyperglycemia relief, and may eventually lead to a 

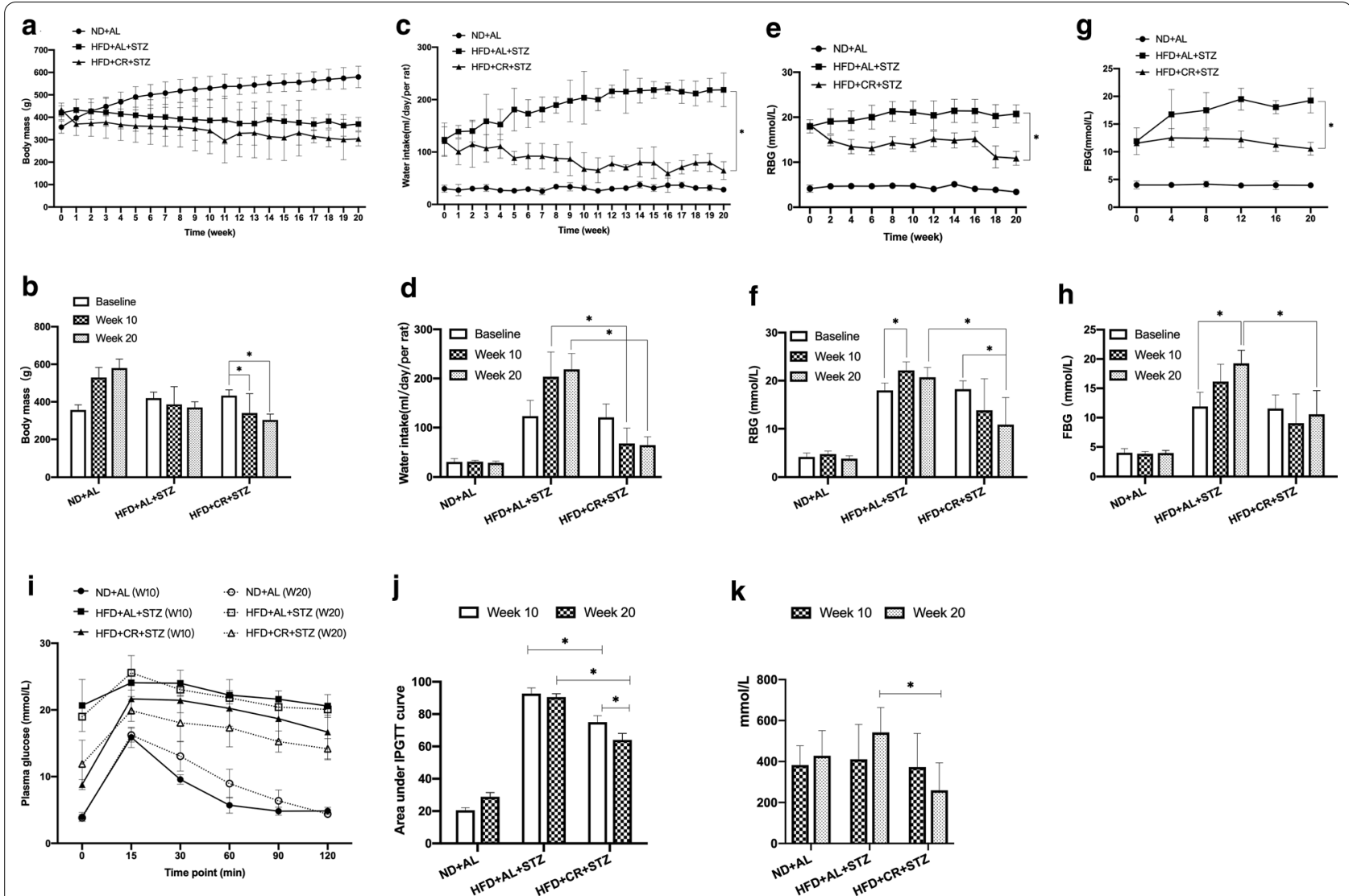

Fig. 2 Effects of CR on metabolic indices. The diabetic Sprague-Dawley rats model was induced by feeding HFD for 8 weeks, followed by a single STZ injection (30 mg/kg). The rats were either provided HFD ab libitum (HFD + AL + STZ group) or fed HFD with a $30 \%$ restriction regimen (HFD + CR + STZ group). Rats given free access to normal chow diet served as a blank control (ND+AL group). All interventions lasted for 20 weeks. $\mathbf{a}$, b body mass; $\mathbf{c}$, d water intake; e, f RBG; $\mathbf{g}, \mathbf{h}$ FBG; $\mathbf{i}$ IPGTT; $\mathbf{j}$ AUC of IPGTT; $\mathbf{k}$ FFAs. Data were presented as the mean \pm standard deviation (SD). For $\mathbf{a}-\mathbf{h}, \mathrm{n}=10-20$ for the ND+AL group and $n=6-14$ for the other two groups. Six rats from each group were randomly chosen for the IPGTT experiment and lipid profile detection. ${ }^{*} p<0.05$ between the two groups; $C R$, calorie restriction; HFD, high-fat diet; STZ, streptozotocin; RBG, random blood glucose; FBG, fasting blood glucose; IPGTT, intraperitoneal glucose tolerance test; AUC, area under curve; FFAs, free fat acids; $\mathrm{ND}+\mathrm{AL}$, normal diet provided ad libitum (BCT group); HFD + AL + STZ, HFD provided ad libitum and STZ injection (MCT group); HFD + CR + STZ, HFD with 30\% CR and STZ injection (CR group)

slight (but not significant) decline in body mass when compared with MCT rats.

Glucotoxicity refers to long-term chronic hyperglycemia leading to downregulated insulin gene expression and chronic irreversibly decreased insulin synthesis. Meanwhile, lipotoxicity refers to the toxic effect of high concentrations of fatty acids in circulation on $\beta$ cells [24]. In our study, hyperglycemia was constantly aggravated in the MCT group over time, indicating more and more severe glucotoxicity. Accordingly, the results from GSIS also revealed almost exhausted insulin secretion. Fortunately, 30\% CR markedly reversed the deterioration of hyperglycemia, leading to relief from glucotoxicity and thus protection of islet function. The role of CR in improving the lipid profile has been widely demonstrated in previous studies [25, 26], as well as in this study. High FFAs level is an independent risk factor for T2DM [27,
28]. In vitro studies have shown that FFAs can damage glucose-stimulated insulin secretion in $\beta$ cells and primary islets, and increase $\beta$-cell apoptosis and necrosis $[29,30]$. Our data demonstrated that sharply increased FFAs level induced by HFD feeding and T2DM pathology could be reversed by $30 \% \mathrm{CR}$. The alleviation of lipotoxicity seemed to exert positive effects on $\beta$ cells as GSIS was eventually improved after CR intervention in our study.

The insulin signaling pathway is crucial for normal insulin action. Defects in the transduction or phosphorylation of the amongst signaling molecules such as PI3K, AKT, and GLUT4 may be relate to the onset of IR. Our data indicated that AKT/AS160/GLUT4 signaling in skeletal muscle was consecutively downregulated with the progression of diabetes and HFD feeding, while CR intervention seemed to exert limited protective effects. Although the expression of AKT, p-AKT, AS160, and 

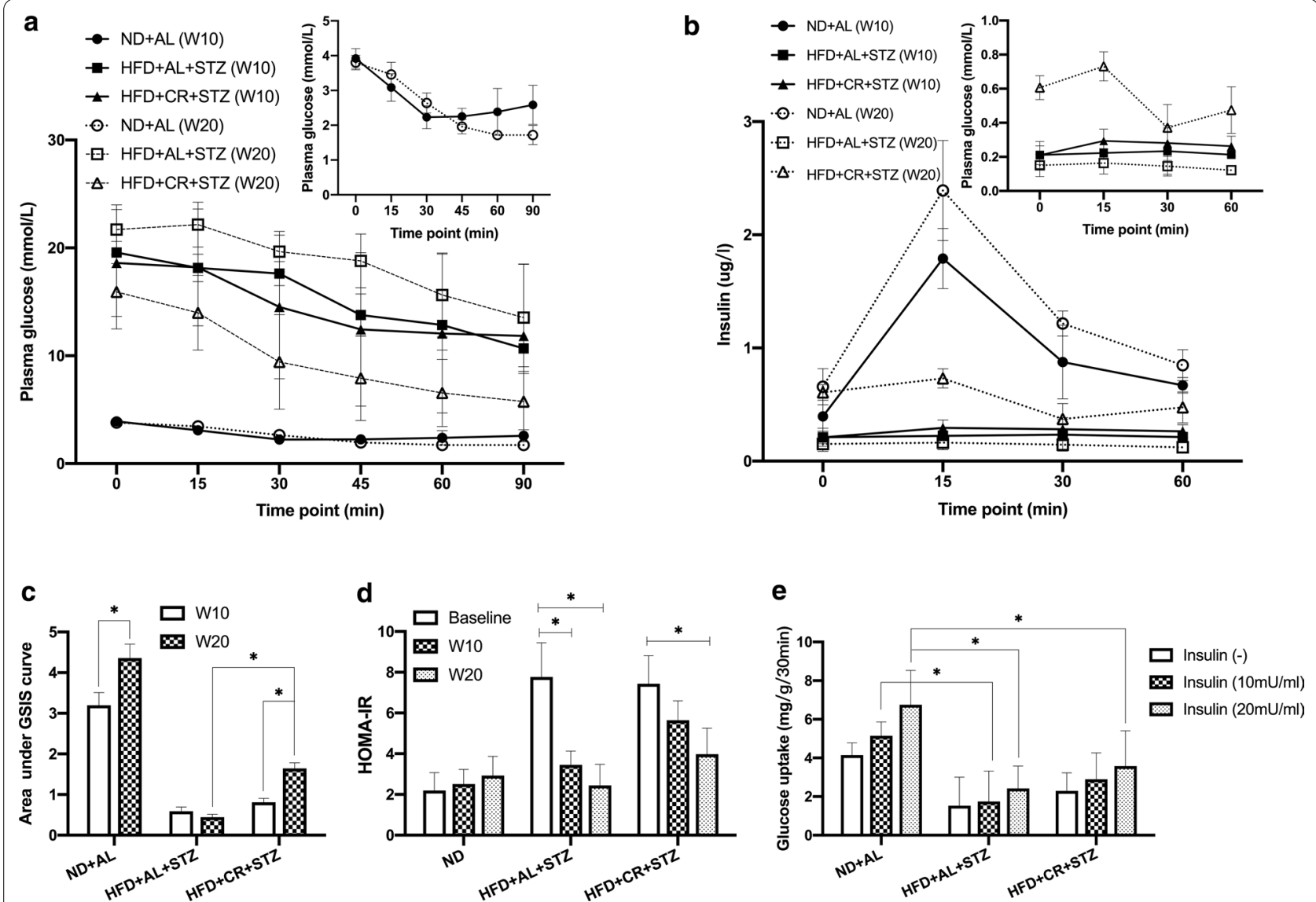

Fig. 3 Effects of CR on insulin resistance and islets function. The diabetic Sprague-Dawley rats model was induced by feeding HFD for 8 weeks, followed by a single STZ injection (30 mg/kg). The rats were either provided HFD ab libitum (HFD + AL + STZ group) or fed HFD with a 30\% restriction regimen (HFD $+C R+S T Z$ group). Rats given free access to normal chow diet served as a blank control (ND+AL group). All interventions lasted for 20 weeks. a ITT $(n=6) ; \mathbf{b}$ GSIS $(n=6)$; c AUC of GSIS; $\mathbf{d}$ HOMA-IR $(n=6-10)$; e glucose uptake in muscle $(n=4)$; data were presented as the mean \pm standard deviation (SD). ${ }^{*} p<0.05$ between the two groups; $C R$, calorie restriction; HFD, high-fat diet; STZ, streptozotocin; ITT, insulin tolerance test; GSIS, glucose simulate insulin secretion; AUC, area under curve; HOMA-IR, homeostasis model assessment of insulin resistance; IOD, integrated optical density; ND + AL, normal diet provided ad libitum (BCT group); HFD + AL + STZ, HFD provided ad libitum and STZ injection (MCT group); HFD + CR + STZ, HFD with 30\% CR and STZ injection (CR group)

GLUT4 proteins was restored to some extent by $30 \%$ $\mathrm{CR}$ at 10 weeks, their expression declined to the levels observed in MCT rats at 20 weeks. In accordance with this, the improvement in glucose uptake induced by $30 \%$ CR was not significant. These results probably suggest that the overall IR improvement induced by $30 \%$ CR may not be due to the alleviation of IR in muscle. This needs to be further confirmed in the future studies. An in vitro study [31] found no significant change in basal glucose uptake rate of skeletal muscle tissue treated with medium containing glucose and insulin equivalent to the $\mathrm{CR}$ level in vivo when compared with the control group.
Mechanism analysis detected no notable changes in p-AKT, AS160, GLUT1, and GLUT4, which was similar to our results. In visceral white adipose tissue, the protein expression levels of AKT, p-AKT, AS160 and GLUT4 in the MCT group decreased progressively over time, which was consistent with the downregulation of insulin signaling in IR individuals reported by many studies. However, these effects could be significantly reversed by $30 \% \mathrm{CR}$ intervention, suggesting the potential of $30 \% \mathrm{CR}$ in delaying impairment of insulin signaling in white adipose tissue during the process of T2DM. 


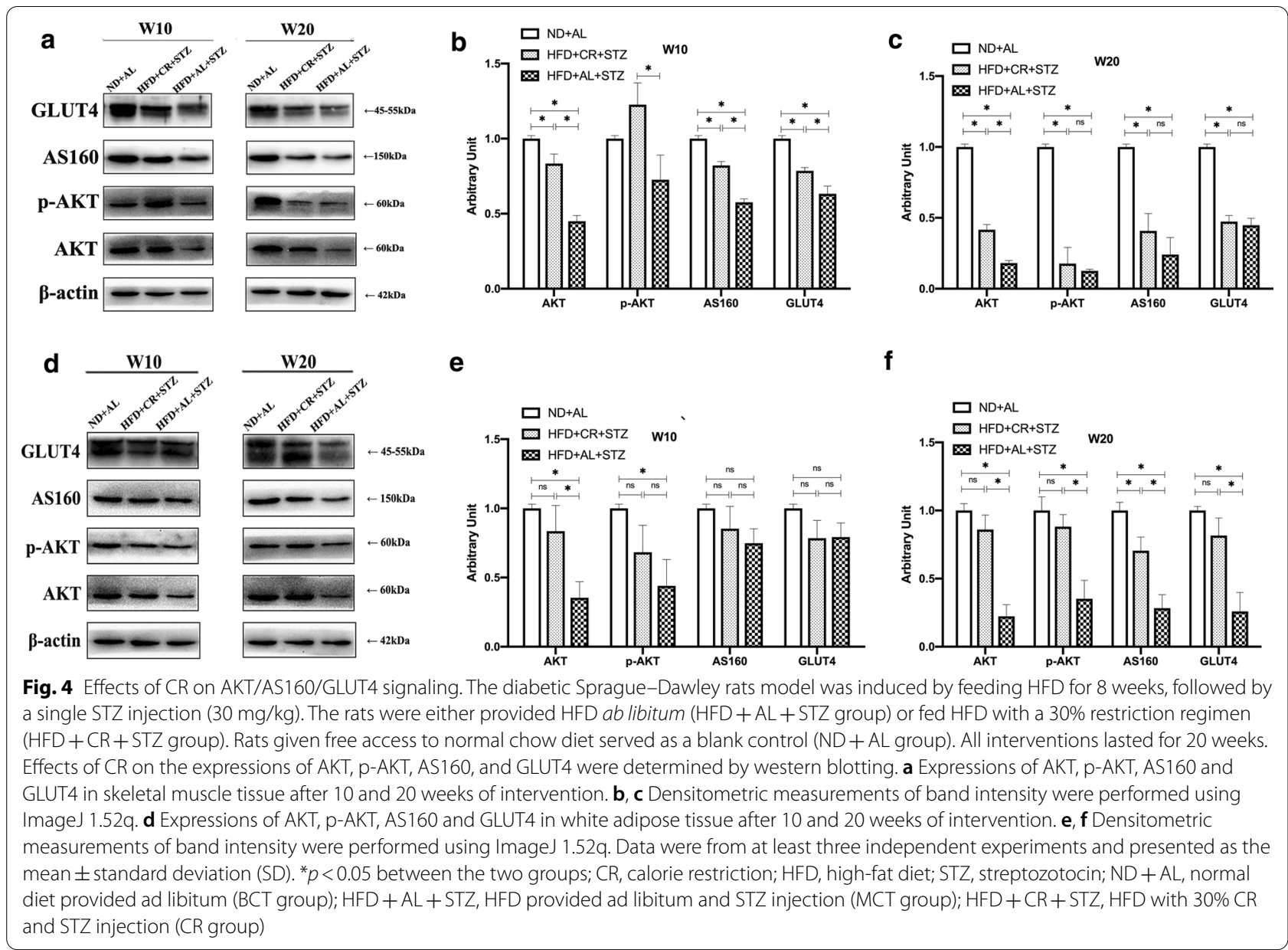

In addition, it was suggested [32] that the remaining $\beta$ cells may proliferate after the destruction of STZ, resulting in spontaneous recovery from hyperglycemia. Hence, diabetic rats were fed HFD to maintain model stability in our experiment. However, overt glucolipotoxicity induced by consecutive HFD feeding accelerated islet failure, especially in the MCT group. Then, the decrease in FINS was much greater than the increase in FBG; therefore, the HOMA-IR value of the MCT group calculated with the formula also exhibited a decreasing tendency, contrary to the aggravated IR reflected by the ITT curve.

Admittedly, the implementation of CR with HFD due to the instability of the STZ-induced diabetic model may have greatly weakened the protective effects of CR. This is one of the limitations of our research. In addition, we did not include included more rats or other diabetic animal models, such as transgenic models $(d b / d b, o b / o b)$, to verify the results repeatedly. In the future, transgenic animal models and larger sample sizes should be employed to better interpret the benefits of $\mathrm{CR}$. The protective effects of CR under the condition of blocked AKT/ AS160/GLUT4 signaling have not been explored yet, and further studies are warranted to consolidate our findings.

\section{Conclusions}

In conclusion, our study demonstrated that during the progression of $\mathrm{T} 2 \mathrm{DM}, 30 \%$ CR could exert protective effects on islet function through significant alleviation of glucolipotoxicity, reflected by markedly improved hyperglycemia and dyslipidemia, and ameliorate insulin resistance. The underlying molecular mechanism are likely related to the upregulation of AKT/AS160/GLUT4 signaling in white adipose tissue. Further studies associated with of AKT/AS160/GLUT4 signaling need to be conducted to enhance the credibility of our results. 


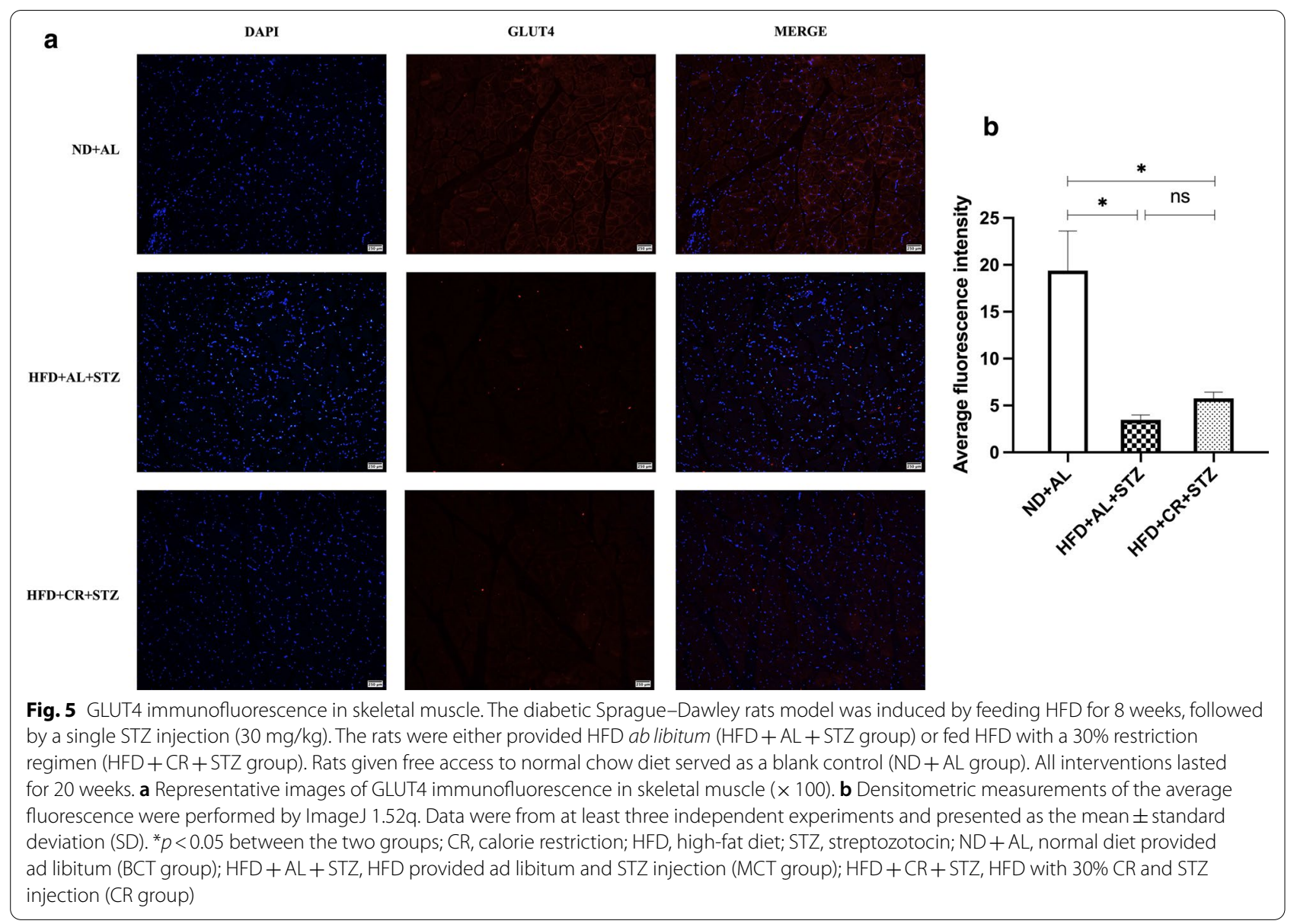

\section{Abbreviations}

T2DM: Type 2 diabetes mellitus; CR: Calorie restriction; HFD: High-fat diet; IR: Insulin resistance; INSR: Insulin receptor; IRS: Insulin receptor substrate; PI3K: Phosphoinositide-3-kinase; AKT: Protein kinase B; AS160: AKT substrate of 160 kDa; GLUT4: Glucose transporter 4; STZ: Streptozotocin; RBG: Random blood glucose; FBG: Fasting blood glucose; ITT: Insulin tolerance test; FINS: Fasting insulin; HOMA-IR: Homeostasis model assessment of insulin resistance; IPGTT: Intraperitoneal glucose tolerance test; GSIS: Glucose-stimulated insulin secretion; WAT: White adipose tissue; KRH: Krebs-Henseleit buffer; BSA: Bovine serum albumin; PBS: Phosphate-buffered saline; FFAs: Free fatty acids; TG: Triglyceride; TC: Total cholesterol; HDL-c: High-density lipoprotein cholesterol; LDL-c: Low-density lipoprotein cholesterol.

\section{Supplementary Information}

The online version contains supplementary material available at https://doi. org/10.1186/s12986-021-00575-y.

Additional file 1: Antibodies used in the experiments and changes in lipid profile post-treatment.

\section{Acknowledgements}

Not applicable.

\section{Authors' contributions}

$J Q, L Z$, and YJH conceived and designed the experiments. LZ, YJH, JPS, TYZ, and TLL performed the study. LZ, YJH, and BK drafted the manuscript. XFS, HL,
GPZ, and ZYY analyzed the data. JQ, BK, and XFS revised the manuscript critically for important intellectual content, clarity, and grammar. All authors read and approved the final manuscript.

\section{Funding}

This study was supported by the National Natural Science Foundation of China (No.81874498), Shenzhen Healthcare Research Project (No. SZLY2018002), Science and Technology Program of Guangzhou (No. 201903010044), and Traditional Chinese Medicine Research Project of Guangming District (No.GM2019020022).

\section{Availability of data and materials}

The datasets used and/or analyzed during the current study are available from the corresponding author upon reasonable request.

\section{Declarations}

\section{Ethics approval and consent to participate}

The animal experiments were performed according to internationally followed ethical standards and approved by the research ethics committee of the School of Public Health, Sun Yat-Sen University (NO. 2019-001).

\section{Consent for publication}

Not applicable.

\section{Competing interests}

The authors declare that they have no competing interests. 


\section{Author details}

${ }^{1}$ Department of Traditional Chinese Medicine, The Seventh Affiliated Hospital, Sun Yat-Sen University, Shenzhen 518107, China. ${ }^{2}$ Department of Traditional Chinese Medicine, The First Affiliated Hospital, Sun Yat-Sen University, Guangzhou 518100, China. ${ }^{3}$ Department of VIP Ward, Sun Yat-Sen University Cancer Center, Guangzhou 510080, China. ${ }^{4}$ Department of Obese and Metabolic Disease, Guangzhou Panyu Hospital of Chinese Medicine, Guangzhou 511400, China

Received: 10 October 2020 Accepted: 16 April 2021

Published online: 05 May 2021

\section{References}

1. Simpson RW, Shaw JE, Zimmet PZ. The prevention of type 2 diabeteslifestyle change or pharmacotherapy? A challenge for the 21st century. Diabetes Res Clin Pract. 2003;59(3):165-80.

2. International Diabetes Federation Diabetes Atlas, 9th edition, IDF, Brussels. https://diabetesatlas.org/upload/resources/2019/IDF_Atlas_9th_ Edition_2019.pdf

3. Lean MEJ, Leslie WS, Barnes AC, et al. Primary care-led weight management for remission of type 2 diabetes (DiRECT): an open-label, clusterrandomised trial. Lancet. 2018:391(10120):541-51.

4. Petersen MC, Shulman GI. Mechanisms of insulin action and insulin resistance. Physiol Rev. 2018;98(4):2133-223.

5. Storgaard $\mathrm{H}$, Song $X M$, Jensen $C B$, et al. Insulin signal transduction in skeletal muscle from glucose-intolerant relatives of type 2 diabetic patients [corrected]. Diabetes. 2001:50(12):2770-8.

6. Pratipanawatr W, Pratipanawatr T, Cusi K, et al. Skeletal muscle insulin resistance in normoglycemic subjects with a strong family history of type 2 diabetes is associated with decreased insulin-stimulated insulin receptor substrate-1 tyrosine phosphorylation. Diabetes. 2001;50(11):2572-8.

7. Tonks KT, Ng Y, Miller S, et al. Impaired Akt phosphorylation in insulinresistant human muscle is accompanied by selective and heterogeneous downstream defects. Diabetologia. 2013;56(4):875-85.

8. Abel ED, Peroni O, Kim JK, et al. Adipose-selective targeting of the GLUT4 gene impairs insulin action in muscle and liver. Nature. 2001:409(6821):729-33.

9. Zisman A, Peroni OD, Abel ED, et al. Targeted disruption of the glucose transporter 4 selectively in muscle causes insulin resistance and glucose intolerance. Nat Med. 2000;6(8):924-8.

10. Moltke I, Grarup N, Jorgensen ME, et al. A common Greenlandic TBC1D4 variant confers muscle insulin resistance and type 2 diabetes. Nature. 2014;512(7513):190-3.

11. LiT, Chen K, Liu G, et al. Calorie restriction prevents the development of insulin resistance and impaired lipid metabolism in gestational diabetes offspring. Pediatr Res. 2017:81(4):663-71.

12. Prasannarong M, Vichaiwong K, Saengsirisuwan V. Calorie restriction prevents the development of insulin resistance and impaired insulin signaling in skeletal muscle of ovariectomized rats. Biochim Biophys Acta. 2012;1822(6):1051-61.

13. Wang $H$, Sharma $N$, Arias $E$, et al. Insulin signaling and glucose uptake in the soleus muscle of 30-month-old rats after calorie restriction with or without acute exercise. J Gerontol Ser A Biol Sci Med Sci. 2015;71(3):323-32.

14. Wheatley KE, Nogueira LM, Perkins SN, et al. Differential effects of calorie restriction and exercise on the adipose transcriptome in diet-induced obese mice. J Obes. 2011;2011:265417.

15. Park SY, Choi GH, Choi HI, et al. Calorie restriction improves whole-body glucose disposal and insulin resistance in association with the increased adipocyte-specific GLUT4 expression in Otsuka Long-Evans Tokushima Fatty rats. Arch Biochem Biophys. 2005;436(2):276-84
16. Xu W, Lu Z, Wang X, et al. Gynura divaricata exerts hypoglycemic effects by regulating the PI3K/AKT signaling pathway and fatty acid metabolism signaling pathway. Nutr Diabetes. 2020;10(1):31.

17. Reed MJ, Meszaros K, Entes $L$, et al. A new rat model of type 2 diabetes: the fat-fed, streptozotocin-treated rat. Metabolism. 2000:49(11):1390-4.

18. Yao H, Gu J, Shan Y, et al. Type 2 diabetes mellitus decreases systemic exposure of clopidogrel active metabolite through upregulation of P-glycoprotein in rats. Biochem Pharmacol. 2020;180:114142.

19. Nambirajan G, Karunanidhi K, Ganesan A, et al. Evaluation of antidiabetic activity of bud and flower of Avaram Senna (Cassia auriculata L.) In high fat diet and streptozotocin induced diabetic rats. Biomed Pharmacother. 2018;108:1495-506.

20. Koshinaka K, Sano A, Howlett KF, et al. Effect of high-intensity intermittent swimming on postexercise insulin sensitivity in rat epitrochlearis muscle. Metab Clin Exp. 2008;57(6):749-56.

21. Chukwuma Cl, Islam MS. Effects of xylitol on carbohydrate digesting enzymes activity, intestinal glucose absorption and muscle glucose uptake: a multi-mode study. Food Funct. 2015;6(3):955-62.

22. Ajiboye BO, Oyinloye BE, Agboinghale PE, et al. Cnidoscolus aconitifolius (Mill.) I. M. Johnst leaf extract prevents oxidative hepatic injury and improves muscle glucose uptake ex vivo. J Food Biochem. 2019:43(12):e13065.

23. Baskota A, Li S, Dhakal N, et al. Bariatric surgery for type 2 diabetes mellitus in patients with $\mathrm{BMl}<30 \mathrm{~kg} / \mathrm{m} 2$ : a systematic review and metaanalysis. PLOS ONE. 2015;10(7):e0132335.

24. Perry RJ, Peng L, Cline GW, et al. Mechanisms by which a very-low-calorie diet reverses hyperglycemia in a rat model of type 2 diabetes. Cell Metab. 2018;27(1):210.e3-17.e3.

25. van Raalte DH, Diamant M. Glucolipotoxicity and beta cells in type 2 diabetes mellitus: Target for durable therapy? Diabetes Res Clin Pract. 2011;93:S37-46.

26. Holowko J, Michalczyk MM, Zajac A, et al. Six weeks of calorie restriction improves body composition and lipid profile in obese and overweight former athletes. Nutrients. 2019;11(7):1461

27. Kraus WE, Bhapkar M, Huffman KM, et al. 2 years of calorie restriction and cardiometabolic risk (CALERIE): exploratory outcomes of a multicentre, phase 2, randomised controlled trial. Lancet Diabetes Endocrinol. 2019;7(9):673-83.

28. Cnop M, Welsh N, Jonas JC, et al. Mechanisms of pancreatic beta-cell death in type 1 and type 2 diabetes - many differences, few similarities. Diabetes. 2005;54:597-107.

29. Paolisso G, Tataranni PA, Foley JE, et al. A high concentration of fasting plasma non-esterified fatty acids is a risk factor for the development of NIDDM. Diabetologia. 1995;38(10):1213-7.

30. Poitout $V$, Robertson RP. Minireview: secondary beta-cell failure in type 2 diabetes - a convergence of glucotoxicity and lipotoxicity. Endocrinology. 2002;143(2):339-42.

31. Arias EB. Cartee GD In vitro simulation of calorie restriction-induced decline in glucose and insulin leads to increased insulin-stimulated glucose transport in rat skeletal muscle. Am J Physiol Endocrinol Metab. 2007;293(6):E1782-8.

32. Su EN, Alder VA, Yu DY, et al. Continued progression of retinopathy despite spontaneous recovery to normoglycemia in a long-term study of streptozotocin-induced diabetes in rats. Graefes Arch Clin Exp Ophthalmol. 2000;238(2):163-73.

\section{Publisher's Note}

Springer Nature remains neutral with regard to jurisdictional claims in published maps and institutional affiliations. 